\title{
Mesenchymal stem cell therapy in osteoarthritis: advanced tissue repair or intervention with smouldering synovial activation?
}

\author{
Peter LEM van Lent* and Wim B van den Berg \\ See related research by Desando et al., http://arthritis-research.com/content/15/1/R22
}

\begin{abstract}
Although it is generally accepted that osteoarthritis is a degenerative condition of the cartilage, other tissues such as synovium in which immunological and inflammatory reactions occur contribute to the development of joint pathology. This sheds new light on the potential mechanism of action of mesenchymal stem cell therapy in osteoarthritis. Rather than tissue repair due to local transformation of injected mesenchymal stem cells to chondrocytes and filling defects in cartilage, such treatment might suppress synovial activation and indirectly ameliorate cartilage damage. Desando and co-workers report in Arthritis Research \& Therapy that intra-articular delivery of adipose-derived stem cells attenuates progression of synovial activation and joint destruction in osteoarthritis in an experimental rabbit model. Clinical studies are warranted to see whether this approach might be a novel way to combat development of joint destruction in inflammatory subtypes of osteoarthritis.
\end{abstract}

\section{Osteoarthritis and smouldering synovitis}

Although it is generally accepted that the primary effect of stem cell treatment occurs through tissue-specific differentiation, new data suggest that the therapeutic potential of these cells might also be related to their paracrine effect $[1,2]$.

Clinically, patients with osteoarthritis (OA) express variable synovitis. Thickening of the lining layer containing predominantly macrophages that exhibit an activated phenotype is found within a substantial subpopulation of OA patients. Synovial macrophages produce

*Correspondence: P.vanlent@reuma.umcn.nl

Rheumatology Research and Advanced Therapeutics, Department of Rheumatology, Radboud University Medical Centre, Nijmegen, The Netherlands elevated levels of pro-inflammatory factors like cytokines (IL-1), complement factors and damage-associated molecular pattern molecules (DAMPs;S100A8/A9) [3].

Release of cartilage matrix fragments from damaged cartilage may give a prolonged stimulation of synovial macrophages, thereby forming a positive feedback loop that drives smouldering synovitis. Previous studies have shown that selective elimination of synovial macrophages prior to induction of experimental murine OA prevented synovial activation and development of joint destruction like cartilage destruction and new formation of cartilage/ bone (osteophytes). Synovial macrophages may contribute to cartilage destruction by prolonged production of pro-inflammatory cytokines or to new formation of osteophytes by release of growth factors [4].

\section{Adipose-derived stem cells express anti-inflammatory characteristics}

Adipose tissue hosts multipotent stem cells that can easily be purified by digestion and adhesion to plastic surfaces and shares numerous properties with bone marrowderived mesenchymal stem cells (MSCs). Five percent of the nucleated fraction in adipose tissue represents stem cells, which is far more than found in adult human bone marrow. Adipose-derived stem cells (ADSCs) have been shown to exhibit immune suppressive properties and release anti-inflammatory molecules like IL-10, IL-1 receptor antagonist (IL-1 ra), indoleamine 2,3-dioxygenase, transforming growth factor (TGF) $\beta$ and prostaglandin E2 [5]

Desando and colleaques [1] induced OA through bilateral anterio cruciate ligament transsection, which causes thickening of the lining layer. A single local administration of ADSCs into such an OA knee joint attenuated inflammation in the synovial membrane and was reflected by less thickening of the lining layer and lowering of matrix metalloproteinase-1 expression. Interestingly, labelled ADSCs were detected within the synovial layer in close contact with synovial macrophages. 
In recent studies it was found that MSCs express an elevated immunosuppressive action when applied to inflammatory regions, suggesting that factors released during inflammation do interact with stem cells, driving their immunosuppressive capacity. Up till now the immune modulatory properties have been clearly detected after treatment of acute graft versus host disease and autoimmune diseases. First, the focus was on $\mathrm{T}$ cells but later on it was found that MSCs also block the generation of functional antigen-presenting cells like macrophages. So MSCs not only inhibit the adaptive immune response but also cells of innate immunity, including macrophages [5].

MSCs can induce polarization towards 'alternative activated' M2 cells characterized by a high expression of CD206 and release of cytokines like IL-10 and lower IL-12 and TNF $\alpha$ production. The immunomodulatory function of ADSCs is strongly elevated by cytokines such as interferon- $\gamma$, TNF $\alpha$ and IL- $1 \alpha / \beta$, which are produced by activated macrophages. These cytokines strongly elevate the production of suppressive factors [6,7]. The immunosuppressive activity is thus not a constitutive property of MSCs but depends on a process of activation or licensing.

\section{Intra-articular deposition of ADSCs protects against joint destruction during experimental OA}

Desando and colleagues further found that a single injection of ADSCs into OA rabbit knee joints protected against development of cartilaginous and meniscal damage. This is in line with a previous study in our lab in a murine osteoarthritis model, where intra-articular injection of collagenase resulted in ligament damage and instability-related OA cartilage damage, along with marked synovial thickening. In that study, injected ADSCs closely interacted with synovial macrophages and inhibited ligament damage and development of joint destruction at later time-points [8]. Apart from inhibition of pro-inflammatory cytokines by macrophages, ADSCs may also stimulate macrophages to produce elevated levels of growth factors.

\section{Osteophytes and synovial activation}

During OA, new formation of cartilage/bone (osteophytes) is found in locations at the joint margins. Intraarticular injection of growth factors like TGF $\beta$ or bone morphogenetic protein-2 can mimic this process and show characteristic patterns [9]. Interestingly, when the synovial lining layer was depleted from macrophages prior to injection of TGF $\beta$, osteophyte formation was greatly reduced, suggesting that TGF $\beta$ acts via activation of macrophages [10]. So, ADSCs may inhibit macrophages to produce TGF $\beta$-regulated factors that are crucial for mediating osteophyte formation.
In summary, recent studies performed in animal OA models show that a single local administration of ADSCs into OA joints induces suppression of synovial activation, and ameliorates damage of the ligaments and menisci, thereby inhibiting development of cartilage destruction and new formation of cartilage/bone. The immunosuppressive phenotype of the ADSCs may thereby be driven by pro-inflammatory factors released by the synovium. Recently started studies in OA patients will soon provide an answer to the question of whether this application will also help to prevent joint destruction within the human OA joint. Special attention needs to be given to subtypes of OA bearing an inflammatory component.

\section{Abbreviations}

ADSC, adipose-derived stem cell; IL, interleukin; MSC, mesenchymal stem cell; $\mathrm{OA}$, osteoarthritis; TGF, transforming growth factor; TNF, tumour necrosis factor.

\section{Competing interests}

The authors declare that they have no competing interests.

Published: 20 March 2013

\section{References}

1. Desando G, Cavallo C, Sartoni F, Martini L, Parrilli A, Veronesi F, Fini M, Giardino R, Facchini A, Grigolo B: Intra-articular delivery of adipose derived stromal cells attenuates osteoarthritis progression in an experimental rabbit model. Arthritis Res Ther 2013, 15:R22.

2. Jorgensen C: Mesenchymal stem cells immunosuppressive properties: is it specific to bone marrow-derived cells? Stem Cell Res Ther 2010, 1:15.

3. Kapoor M, Martel-Pelletier J, Lajeunesse D, Pelletier JP, Fahmi H: Role of proinflammatory cytokines in the pathophysiology of osteoarthritis. Nat Rev Rheumatol 2011, 7:33-42.

4. Blom AB, van Lent PL, Holthuysen AE, van der Kraan PM, Roth J, van Rooijen $\mathrm{N}$,van den Berg WB: Synovial lining macrophages mediate osteophyte formation during experimental osteoarthritis. Osteoarthritis Cartilage 2004, 12:627-635.

5. Spaggiari GM, Moretta L: Cellular and molecular interactions of mesenchymal stem cells in innate immunity. Immuno/ Cell Biol 2013, 91:27-31.

6. Maggini J, Mirkin G, Bognanni I, Holmberg J, Piazzón IM, Nepomnaschy I, Costa H, Cañones C, Raiden S, Vermeulen M, Geffner JR: Mouse bone marrow-derived mesenchymal stromal cells turn activated macrophages into a regulatory-like profile. PLoS One 2010, 5:e9252.

7. Crop MJ, Baan CC, Korevaar SS, ljzermans JN, Pescatori M, Stubbs AP, van ljcken WF, Dahlke MH, Eggenhofer E, Weimar W, Hoogduijn MJ: Inflammatory conditions affect gene expression and function of human adipose tissuederived mesenchymal stem cells. Clin Exp Immunol 2010, 162:474-486.

8. ter Huurne M, Schelbergen R, Blattes R, Blom A, de Munter W, Grevers LC,Jeanson J, Noël D, Casteilla L, Jorgensen C, van den Berg W, van Lent PL: Antiinflammatory and chondroprotective effects of intraarticular injection of adipose-derived stem cells in experimental osteoarthritis. Arthritis Rheum 2012, 64:3604-3613.

9. Van der Kraan PM, van den Berg WB: Osteophytes: relevance and biology. Osteoarthritis Cartilage 2007, 15:237-244.

10. van Lent $\mathrm{PL}, \mathrm{Blom} A B$, van der Kraan $\mathrm{P}$, Holthuysen $A E$, Vitters $E$, van Rooijen $N$, Smeets RL, Nabbe KC, van den Berg WB: Crucial role of synovial lining macrophages in the promotion of transforming growth factor betamediated osteophyte formation. Arthritis Rheum 2004, 50:103-111.

\section{doi:10.1186/ar4190}

Cite this article as: van Lent PLEM, ven den Berg WB: Mesenchymal stem cell therapy in osteoarthritis: advanced tissue repair or intervention with smouldering synovial activation? Arthritis Research \& Therapy 2013, 15:112. 